\title{
Endoscopic treatment of gastric antral vascular ectasia in real-life settings: argon plasma coagulation or endoscopic band ligation?
}

Running head: Endoscopic treatment of GAVE syndrome

Anna FÁBIÁN ${ }^{1}$; Renáta BOR ${ }^{1}$; Ella SZABÓ1; Viktor KARDOS ${ }^{1}$, Anita BÁLINT ${ }^{1}$; Klaudia FARKAS $^{1}$; Ágnes MILASSIN ${ }^{1}$, Mariann RUTKA ${ }^{1}$, Kata SZÁNTÓ ${ }^{1}$, Tamás MOLNÁR ${ }^{1}$; Mónika SZÜCS²; Katalin LÖRINCZY³; Ákos ORBÁN-SZILÁGYI³, Tibor GYÖKERES³; György GYIMESI ${ }^{4}$; Attila SZEPES(†) ${ }^{4}$; Valéria KOVÁCS ${ }^{5}$; István RÁCZ ${ }^{5}$; Zoltán SZEPES ${ }^{1}$

${ }^{1}$ University of Szeged, First Department of Medicine, 6720 Szeged, Korányi fasor 8-10,

Hungary

${ }^{2}$ University of Szeged, Department of Medical Physics and Informatics, 6720 Szeged,

Korányi fasor 9, Hungary

${ }^{3}$ Hungarian Defence Forces Medical Centre, Department of Gastroenterology, 1062 Budapest,

Podmaniczky u. 11, Hungary

${ }^{4}$ Bács-Kiskun County University Teaching Hospital, Department of Gastroenterology, 6000

Kecskemét, Nyíri u. 38, Hungary

${ }^{5}$ Petz Aladár County Teaching Hospital, Internal Medicine Department and Gastroenterology, 9023 Győr, Vasvári Pál u. 2-4, Hungary

This article has been accepted for publication and undergone full peer review but has not been through the copyediting, typesetting, pagination and proofreading process which may lead to differences between this version and the Version of Record. Please cite this article as doi: $10.1111 / 1751-2980.12958$ 
Correspondence to: Anna Fábián, MD

First Department of Medicine, University of Szeged, Szeged

6720 Szeged, Korányi fasor 8-10, Hungary

Email: fabiananna9@gmail.com

Telephone: +36 62545 186, Fax: +36 62545185

Results from the study were presented as an oral presentation at the United European

Gastroenterology Week 2016. For reference see:

Fabian A, Bor R, Szabo E et al. Endoscopic treatment of gastric antral vascular ectasia: a retrospective multicentre clinical study. UEGW; A134. UEGJ 4 (5S). 2016.

Author contributions: Anna Fábián, Renáta Bor, Ella Szabó and Zoltán Szepes designed the study; Tamás Molnár, Tibor Gyökeres, György Gyimesi, Attila Szepes, István Rácz and Zoltán Szepes performed the endoscopic examinations; Viktor Kardos, Anita Bálint, Klaudia Farkas, Mariann Rutka, Ágnes Milassin, Kata Szántó, Katalin Lőrinczy, Ákos Orbán-Szilágyi, György Gyimesi and Valéria Kovács collected and analyzed the data; Mónika Szücs performed the statistical analysis; Anna Fábián wrote the paper; Tamás Molnár, Tibor Gyökeres, Attila Szepes, István Rácz and Zoltán Szepes provided critical revision.

\section{Abstract}


Aim: Efficacy of argon plasma coagulation (APC) in gastric antral vascular ectasia (GAVE) impairs on long term and depends highly on application settings. Endoscopic band ligation (EBL) might be an alternative, but evidence is limited. This study aimed to evaluate and compare the clinical efficacy of APC and EBL in GAVE.

Methods: Changes in hemoglobin and transfusion need, number of treatment sessions and hospitalizations were retrospectively assessed in 63 transfusion-dependent GAVE patients (mean age: 67.1 years, 54\% female) treated with either APC or EBL (45 and 18 patients, respectively) in four tertiary endoscopic centers.

Results: Both methods increased hemoglobin levels and decreased transfusion need substantially $(2.20 \pm 0.40 \mathrm{~g} / \mathrm{dl}$ and $-5.62 \pm 2.30$ blood units with APC, and $2.74 \pm 0.61 \mathrm{~g} / \mathrm{dl}$ and 4.79 \pm 2.46 blood units with EBL), without significant difference between the methods.

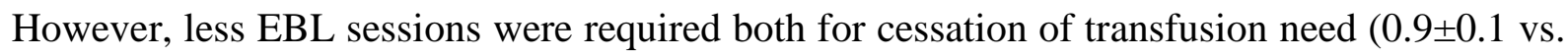
$1.69 \pm 0.31, \mathrm{p}=0.028$ ) and for the resolution of GAVE lesions (2.86 \pm 0.68 vs. $4.43 \pm 0.76$, $\mathrm{p}=0.126)$.

Conclusions: Both APC and EBL are effective in GAVE treatment. EBL might be superior in terms of number of treatment sessions, but not the influence on hemoglobin level and transfusion need. Further prospective studies with homogenized large case number and standardized APC settings are needed.

\section{Keywords}


gastric antral vascular ectasia; endoscopic treatment; argon plasma coagulation; endoscopic band ligation; efficacy

\section{Introduction}

Gastric antral vascular ectasia (GAVE) is a rare, but potentially severe cause of recurrent, upper gastrointestinal bleeding in the elderly. Although occasionally acute gastrointestinal hemorrhage can be observed, most patients present with occult blood loss and chronic, irondeficiency anemia; 60-70\% of them are transfusion-dependent. ${ }^{1}$ Recurrent hospitalizations associated with transfusions might as well be a burden to the healthcare system, not only to patients.

GAVE is characterized by mucosal and submucosal vascular ectasias presenting endoscopically as red, spot-like lesions, arranged in linear strips converging at the pylorus or appear diffusely, located predominantly in the gastric antrum. Two morphological types have been reported: the linear type is more commonly seen in non-cirrhotic patients with female predominance and at an older age compared to the diffuse type that tends to occur in cirrhotic patients with male predominance. ${ }^{2-6}$

Owing to the high risk of surgery and systemic side effects and limited success of pharmacological therapies, endoscopic procedures are favorable in the treatment of GAVE. ${ }^{4}$ The non-contact thermal coagulation technique, argon plasma coagulation (APC) is considered the gold standard (Figure 1/A), even though its long-term efficacy is questionable, and evidence may not be strong enough to support its exclusive role in GAVE. ${ }^{4-8}$ Although various APC 
settings enable a variety of clinical applications, inappropriate definition of applicable settings may lead to impaired efficiency and adverse events. ${ }^{9-10}$ Endoscopic band ligation (EBL) seems to be a comparable and promising alternative (Figure 1/B) with the ability to treat deeper mucosal and submucosal lesions as well in a uniform manner; it might even be superior to APC in terms of required treatment sessions..$^{4,7,11-13}$ It is accessible in most centers and is considered a relatively easy procedure to perform. ${ }^{14-15}$ Results of radiofrequency ablation (RFA) which also offers uniform ablation depth are encouraging as well, although usually further training is required to familiarize with the technique. ${ }^{16-17}$ According to a recent meta-analysis, RFA required fewer treatment sessions than APC and resulted in fewer and less severe complications. ${ }^{18}$ The lack of recommendations regarding the use of either method is a significant limiting factor in the treatment of GAVE.

This retrospective, multicenter study aimed to evaluate and compare the clinical efficacy of APC and EBL in real-life settings.

\section{Methods}

\section{Study design}

Consecutive transfusion-dependent GAVE patients treated with either APC or EBL at any of the four tertiary endoscopic centers involved during a 10-year period were included in this study. Patients in whom other source of gastrointestinal bleeding was revealed throughout the study period were excluded. Both methods were first treatment choices (based on individual consideration of the examiner and local preferences), none of the involved patients treated with EBL underwent previous APC treatment, and vice versa. APC (Figure 2) was performed with 
a MAJ-977 Olympus ENDOPLASMA unit and an EMED endo unit, using one of the following power settings: $30 \mathrm{~W}, 50 \mathrm{~W}$ or $70 \mathrm{~W}$, and a gas flow setting of $2.4 \mathrm{~L} / \mathrm{min}$. EBL (Figure 3) was performed with a standard ligation set carrying 6 ligation bands. In the lack of uniform recommendations, APC settings, application time, size of the treated area, and the number of rubber bands applied with EBL were based on individual consideration. An informed consent was signed by all patients before the endoscopic procedures. In our clinical practice, follow-up endoscopy was performed 4-6 weeks after the first treatment session to check the regression of GAVE lesions and the necessity and timing of further endoscopic treatment. Hemoglobin values were measured monthly and transfusion need was assessed every 4-6 weeks. After the follow-up endoscopy or repeated treatment session, endoscopic treatment was repeated only in case of persisting transfusion need or evidence of GI bleeding.

\section{Outcome measures}

Number of treatment sessions, treatment duration, pre- and post-treatment hemoglobin values and number of blood transfusions were compared between those underwent APC and EBL. Cessation of transfusion need (i.e. stable hemoglobin levels without blood transfusion every 46 weeks after the last treatment session) was considered the primary end-point, and endoscopic resolution of GAVE lesions (i.e. no lesions requiring intervention can be seen on follow-up endoscopy) was assessed as secondary end-point. Follow-up period was defined as the interval between the last endoscopic treatment procedure and the last follow-up visit (when necessity of further endoscopic treatment was decided based on patients' complaints and laboratory parameters) recorded at the database. Indications for transfusion included the following: in case of acute bleeding, $30 \%$ loss of total blood volume made up a relative indication and $40 \%$ loss 
of total blood volume an absolute indication for blood transfusion. In case of chronic anemia, blood transfusion was indicated when clinical symptoms of anemia related hypoxia occurred (mainly when hemoglobin values dropped below $7 \mathrm{~g} / \mathrm{dl}$ ).

\section{Ethical considerations}

This study was carried out in accordance with the Helsinki Declaration and was approved by the Regional and Institutional Human Medical Biological Research Ethics Committee of University of Szeged (clinical trial registration number: 3680 SZTE, date of approval: 17/11/2015).

\section{Statistical methods}

Statistical data were reported as the mean; with frequencies (n) and percentages (\%), when appropriate. Pearson's chi-squared test or Fisher's exact test was used to analyze categorical data, whereas independent samples t-test was used in case of continuous data. Statistical tests were performed using $R$ statistical software. ( $R$ version 3.1.2), values of $p<0.05$ were considered significant.

\section{Results}

\section{Demographic data}

A total of 63 patients were included in the study (mean patient age: 67.1 years, 54\% female). Thirty-one patients (49\%) presented with diffuse and 32 with linear GAVE (51\%). Overt gastrointestinal bleeding occurred in 24 patients, and occult bleeding resulting in chronic iron deficiency anemia was present in 43 patients. Cirrhosis was present in $43 \%$ of the patients: it 
was more frequent in patients with diffuse GAVE compared to linear one (58\% vs. 28\%). Detailed demographic data are shown in Table 1. Forty-five patients were treated with APC and 18 patients with EBL. In case of APC, higher power settings were preferred based on the available data (70 W in 21 cases and $50 \mathrm{~W}$ in 7 cases vs. only $30 \mathrm{~W}$ in 2 cases; power settings were not reported in 15 cases). In case of EBL, an average of 5.3 rubber bands was applied per treatment session. Eleven patients (17\%) were lost to follow-up; average follow-up period was 15.4 months (range: 1-63 months, median: 11 months).

\section{Outcomes regarding hemoglobin levels, transfusion need and complications}

Both treatment methods resulted in significantly increased hemoglobin levels (Figure 4) and decreased transfusion need (Figure 5). The need for blood transfusions ceased totally in 33 patients (25 treated with APC and 8 treated with EBL), in these patients no more transfusions were needed throughout the follow-up period (13.5 \pm 2.5 months for APC and 18.1 \pm 3.8 months for EBL) after the endoscopic treatments. Neither did any of the patients require antrectomy as an alternative to treat GAVE lesions. Complications occurred in a total of 6 patients: 2 patients were reported to have Mallory-Weiss syndrome and 2 patients had ulcerations without active bleeding resulting in scarring after APC, while hyperplastic polyps developed in 2 patients after EBL.

\section{Number of treatment sessions required}

Cessation of transfusion need required less treatment sessions than eradication of GAVE lesions with both APC and EBL. The difference between the number of treatment sessions required for the different endpoints was significant for patients treated with EBL $(0.9 \pm 0.1$ vs. $1.69 \pm 0.31$ 
treatment sessions for cessation of transfusion need and endoscopic remission, respectively, $\mathrm{p}=0.028)$, but not for those treated with APC (2.86 \pm 0.68 vs. $4.43 \pm 0.76$ treatment sessions for cessation of transfusion need and endoscopic remission, respectively, $\mathrm{p}=0.126$ ).

Diffuse GAVE lesions tended to require more treatment sessions compared to linear ones regarding both methods - especially when choosing cessation of transfusion need as an endpoint (3.24 \pm 0.80 vs. $1.40 \pm 0.63$ sessions, $\mathrm{p}=0.078)$. In terms of endoscopic remission, diffuse and linear GAVE both required an average of 3 treatment sessions $(3.73 \pm 0.81$ vs. 3.41 \pm 0.75 sessions, respectively, $\mathrm{p}=0.774$ ).

\section{Comparison of APC and EBL}

When comparing the two treatment options (Table 2), no significant difference was found in the influence of the methods on hemoglobin level elevation $(2.20 \pm 0.40 \mathrm{~g} / \mathrm{dl}$ for APC vs. 2.74 $\pm 0.61 \mathrm{~g} / \mathrm{dl}$ for EBL, $\mathrm{p}=0.461)$ and transfusion need (5.62 \pm 2.30 units for APC vs. 4.79 \pm 2.46 units for EBL, $\mathrm{p}=0.806$ ). However, significantly less treatment sessions (both for cessation of transfusion need [0.9 \pm 0.1 vs. 2.86 $\pm 0.68, \mathrm{p}=0.007]$ and for resolution of GAVE lesions [1.69 \pm 0.31 vs. $4.43 \pm 0.76, \mathrm{p}=0.002])$ and hospitalizations ( $1.33 \pm 0.33$ vs. $4.28 \pm 0.81, \mathrm{p}=0.002)$ were required in case of EBL.

\section{Optimal method for linear and diffuse GAVE}

Whereas in diffuse GAVE, significantly less treatment sessions were required with EBL both for cessation of transfusion need $(1.00 \pm 0.00$ vs. $3.94 \pm 0.01, \mathrm{p}=0.009)$ and for endoscopic resolution of GAVE lesions (1.25 \pm 0.16 vs. $4.83 \pm 1.08$, $\mathrm{p}=0.004)$; in linear GAVE, no difference was detected between the different treatment methods regarding potential endpoints (i.e. neither 
in terms of cessation of transfusion need [ $1.56 \pm 0.78$ vs. $0.75 \pm 0.25, p=0.335$ ], nor endoscopic resolution of lesions [3.83 \pm 1.01 vs. $2.4 \pm 0.68, \mathrm{p}=0.258])$.

\section{Discussion}

Currently, APC is considered the gold standard treatment of GAVE based on its wide availability, cost-effectiveness, efficacy and safety. 4, 15, 19 Superficial vascular lesions are usually treated with 40-50 $\mathrm{W}$ and $0.8 \mathrm{~L} /$ min considering different tissue sensitivities in various locations. ${ }^{9,} 20$ It should be noted however that variable power (30-100 W) and gas flow (0.8-2 $\mathrm{L} / \mathrm{min}$ ) settings have been reported for hemostasis of bleeding vascular ectasias, and in the lack of comparative studies on individual safety and efficacy profiles, the choice of applicable APC settings should be based on the operator's expertise and experience, not on uniform recommendations. ${ }^{20}$

Clinical (cessation of blood transfusion need) success rate of APC in GAVE varies between 90\% to 94\%, and technical (endoscopic obliteration or histological resolution of GAVE lesions) success rate is between $81 \%$ and $86 \% .{ }^{9,}{ }^{19}$ In the clinical setting, it is undecided which variable should be considered the primary end-point of the treatment. According to Peter, only GAVE lesions with symptoms related to blood loss should be treated. ${ }^{21}$ On the other hand, residual lesions might be the source of further blood loss resulting in recurrent transfusion need on the long term. Our clinical practice tends to reflect the former attitude, and in the lack of unequivocal recommendations follow-up endoscopy was not routinely performed after each treatment session to assess regression of GAVE lesions. Treatment time and number of treatment sessions depend on the morphological pattern (eradication of diffuse GAVE may take 
more treatment sessions), the extent of lesions, and the clinical presentation (active bleeding requires more sessions), as well as individual expertise. ${ }^{3,21}$ A slight difference between the number of treatment sessions required for cessation of transfusion need in diffuse GAVE compared to linear one could also be observed among our patients ( $3.24 \pm 0.80$ vs. $1.40 \pm 0.63$ sessions). However, when choosing endoscopic resolution of lesions as treatment endpoint, no such difference could be detected between the morphological types ( $3.73 \pm 0.81$ vs. $3.41 \pm 0.75$ sessions for diffuse and linear GAVE, respectively, $\mathrm{p}=0.774)$.

Complications associated with APC include subcutaneous emphysema, abdominal pain, development of hyperplastic polyps, chronic ulceration, stricture formation, and perforation. In our cohort, Mallory-Weiss syndrome that required no further intervention and ulcerations leading to scarring developed both in only two patients.

Despite the initial success of APC, recurrence rate of GAVE lesions is high (25-100\%) and the efficacy of the modality impairs in the medium and long term (it can be as low as $40 \%$ ), thus multiple treatment sessions (usually 1-5) are often required. ${ }^{4,}$ 7, 15, 19, 22-23 In our study, 56\% clinical success rate could be obtained with an average of 5 APC sessions. Impaired long-term efficacy might be due to inadequacy of standard APC settings to safely ablate dilated capillaries extending to deeper mucosal layers. ${ }^{20}$ The "gold standard" role of APC in GAVE is not reflected in the 2015 ESGE guideline on the management of nonvariceal upper GI hemorrhage that recommends endoscopic hemostasis therapy (including both APC and EBL) for the management of upper GI angiectasias, but none of them specifically. ${ }^{8}$ 
EBL, that was initially used in cases where APC was not available or as a salvage therapy in case of inefficient APC treatment, became a viable alternative due to its ability to treat larger areas at once in a more uniform manner, and to safely obliterate the submucosal vascular plexus that is supposed to play a part in the recurrence of GAVE-related symptoms. ${ }^{4,21,23}$ Dias de Castro also proposed the possible role of EBL as a first-line treatment of GAVE. ${ }^{15}$ The method is widely available, simple, and the relatively short procedure time makes it easy to tolerate. Only mild adverse events were reported (epigastric pain, ulcers and mild bleeding at the treatment site, fever). ${ }^{3,13}$

The potential superiority of EBL compared to endoscopic thermal therapy was first studied by Wells in 2008. Besides the increased hemoglobin levels, decreased transfusions and hospital admissions compared to endoscopic thermal therapy; fewer treatment sessions were required with EBL (1.9 vs. 4.7). ${ }^{4,19,24-25}$ It should be noted however, that in this study a higher number of treatment sessions and lower efficacy rate of APC was reported than in other studies, possibly reflecting a different cohort choice. ${ }^{4}$

The retrospective comparison study of Sato revealed a higher recurrence rate of liver diseaseassociated GAVE lesions treated with APC compared to EBL (68.2\% vs. 8.3\%). In this setting, patients treated with EBL had more severe GAVE lesions (half of them were previously treated with APC as well), thus the higher number of EBL treatment sessions (2-4) and ligation bands applied (19.3 bands on average) might be contributed to this fact. Even so, authors proposed the beneficial use of EBL in severe, diffuse GAVE. ${ }^{7}$ 
In the prospective study of Abdelhalim, EBL proved to be superior to APC regarding the rate of cessation of bleeding (95\% vs. 65\%), lower recurrence rate during the 6-month follow-up (5\% vs. 40\%), higher increase in hemoglobin ( $\mathrm{p}=0.179)$, less transfusion requirements $(\mathrm{p}<0.01)$, decreased rate of hospitalization $(p=0.045)$, and fewer treatment sessions $(p=0.001)$ in patients with GAVE and accompanying liver cirrhosis. ${ }^{12}$

These findings were also confirmed by Elhendawy in a prospective randomized controlled study involving 88 cirrhotic GAVE patients. Significantly less treatment sessions were required with EBL to achieve endoscopic resolution of the lesions (2.98 vs. 3.48), although both methods increased hemoglobin levels significantly. ${ }^{13}$ It should be mentioned that both studies ${ }^{12-13}$ had relatively short follow-up period (6 months) compared to our study (mean: 18 months, median: 12 months) that may hinder the assessment of long-term outcomes.

More recently, the efficacy of EBL and APC in the treatment of GAVE was also assessed in the pediatric population. The prospective comparison by Ghobrial confirmed the superiority of EBL regarding the number of sessions required for complete obliteration of the lesions $(1.85 \pm 0.81$ vs. $4.15 \pm 1.22, \mathrm{p}<0.05)$, procedure time, transfusion requirement, hospitalization and recurrence rate of GAVE lesions. ${ }^{14}$

Unlike in previous studies, we focused mainly on the clinical success rate of APC and EBL in GAVE, not the technical one. The rate of cessation of bleeding was found to be lower for both methods compared to previous results (56\% for APC and 44\% for EBL). Nevertheless, it should be highlighted that hemoglobin levels stabilized at a significantly higher level with both treatment options, even if transfusion need did not cease totally in some patients (although 
decreased substantially). We would like to highlight the significant discrepancy between intertreatment intervals in our study reflecting real-life settings and the shorter intervals used in prospective studies ${ }^{12-13}$. In the clinical setting, frequently repeated endoscopic interventions without justification by clinical symptoms may be a severe burden to patients. Significantly less treatment sessions and hospitalizations in case of EBL suggest a potential benefit for health facilities with limited financial resources ${ }^{4,7}$, as not only the per treatment cost of APC probes and ligation sets, but the number of treatment sessions and blood transfusions should be considered when calculating healthcare related costs. However, no comparative cost-efficacy studies are available yet.

Although the therapeutic efficacy of the modalities has not been thoroughly investigated in different subtypes of GAVE, APC is still considered the first treatment choice in linear GAVE, but EBL might be beneficial in diffuse and severe GAVE. ${ }^{7,}{ }^{21}$ Even though our findings (less treatment sessions with EBL in diffuse GAVE) are consistent with this, it may not be reflected in case numbers (i.e. APC was performed in most cases) due to variation in local preferences.

\section{Limitations}

Retrospective design was the main limiting factor, especially in case of follow-up. Inhomogeneous case numbers might also result in slight biases just like the individual consideration-based treatment choice. In certain cases, small case numbers were insufficient to provide reliable statistical data. Incomplete reporting of applied APC settings did not allow further investigation regarding the optimal APC settings.

\section{Conclusions}


Currently, there is no consensus on the preferable endoscopic treatment of GAVE. In our multicenter comparative study, which is among those with the highest total case number and longest follow-up period regarding this issue, both APC and EBL proved to be effective. Our results suggest the potential superiority of EBL in terms of number of treatment sessions and hospitalizations, but not in the extent to which the two methods influenced hemoglobin level and transfusion need. Considering healthcare related costs and patient compliance, there is a possibility that EBL might be proposed as an alternative first treatment choice for GAVE, not only an adjunctive method in cases of APC failure. The optimal treatment method in the subentities of GAVE has not been thoroughly investigated in the few prospective studies previously. Our study suggests that EBL might be beneficial in diffuse GAVE. On the other hand, defining more specific APC setups for GAVE may also improve performance and efficacy. Still, there is a pressing need for further prospective studies with homogenized large case number and settings resembling more to real-life settings to establish recommendations about the endoscopic treatment of GAVE.

\section{Acknowledgements}

Declaration of conflicting interests: None declared.

Funding statement: This research received no specific grant from any funding agency in the public, commercial, or not-for-profit sectors. 


\section{References}

1. Garg H, Gupta S, Anand AC, Broor SL: Portal hypertensive gastropathy and gastric antral vascular ectasia. Indian J Gastroenterol 2015; 34(5): 351-358

2. Shah N, Cavanagh Y, Kaswala DH, Shaikh S: Development of hyperplastic polyps following argon plasma coagulation of gastric antral vascular ectasia. J Nat Sci Biol Med. 2015; 6(2): 479-482

3. Zepeda-Gómez S, Marcon NE: Endoscopic band ligation for nonvariceal bleeding: A review. Can J Gastroenterol 2008; 22 (9): 748-752

4. Naidu H, Huang Q, Mashimo H: Gastric antral vascular ectasia: the evolution of therapeutic modalities. Endoscopy International Open 2014; 02: E67-E73

5. Zulli C, Del Prete A, Romano M, Esposito F, Amato MR, Esposito P: Refractory gastric antral vascular ectasia: a new endoscopic approach. European Review for Medical and Pharmacological Sciences. 2015; 19: 4119-4122

6. Fuccio L, Mussetto A, Laterza L, Eusebi LH, Bazzoli F: Diagnosis and management of gastric antral vascular ectasia. World J Gastrointest Endosc 2013; 5(1): 6-13

7. Sato T, Yamazaki K, Akaike J: Endoscopic band ligation versus argon plasma coagulation for gastric antral vascular ectasia associated with liver diseases. Digestive Endoscopy 2012; 24: 237-242

8. Gralnek IM, Dumonceau JM, Kuipers EJ, Lanas A, Sanders DS, Kurien M et al.: Diagnosis and management of nonvariceal upper gastrointestinal hemorrhage: European Society of Gastrointestinal Endoscopy (ESGE) Guideline. Endoscopy 2015; 47(10): 1-46. 
9. Robotis J, Sechopoulos P, Rokkas T: Argon plasma coagulation: Clinical applications in Gastroenterology. Annals of gastroenterology 2003, 16(2): 131-137

10. Asge Technology Committee, Conway JD, Adler DG, Diehl DL, Farraye FA, Kantsevoy SV, Kaul V et al.: Endoscopic hemostatic devices. Gastrointest Endosc. 2009; 69(6): 987-96

11. Zepeda-Gómez S, Sultanian R, Teshima C, Sandha G, Van Zanten S, Montano-Loza AJ: Gastric antral vascular ectasia: a prospective study of treatment with endoscopic band ligation. Endoscopy. 2015; 47(6): 538-40

12. Abdelhalim H, Mostafa I, Abdelbary MS, Elansary M, Abdo M, Rahim AA: Endoscopic Band Ligation Versus Argon Plasma Coagulation for the Treatment of Gastric Antral Vascular Ectasia in Egyptian Patients with Liver Cirrhosis. World Journal of Medical Sciences 2014; 10 (3): 357-361

13. Elhendawy M, Mosaad S, Alkhalawany W, Abo-Ali L, Enaba M, Elsaka A et al.: Randomized controlled study of endoscopic band ligation and argon plasma coagulation in the treatment of gastric antral and fundal vascular ectasia. United European Gastroenterol J. 2016; 4(3): 423-428

14. Ghobrial C, Rabea M, Mohsen N, Eskander A: Gastric antral vascular ectasia in portal hypertensive children: Endoscopic band ligation versus argon plasma coagulation. $J$ Pediatr Surg. 2018 Jul 27

15. Dias de Castro F, Carvalho PB, Goncalves TC, Magalhaes J, Moreira MJ, Marinho C et al.: Treating Gastric Antral Vascular Ectasia - When Argon Therapy Is Not Enough GE Port J Gastroenterol. 2016; 23(5): 249-253 
16. Zepeda-Gomez S: Endoscopic treatment of gastric antral vascular ectasia: current opinions. GE Port J Gastroenterol. 2017; 24(4): 176-182

17. St Romain P, Boyd A, Zheng J, Chow SC, Burbridge R, Wild D: Radiofrequency ablation (RFA) vs. Argon plasma coagulation (APC) for the management of gastric antral vascular ectasia (GAVE) in patients with and without cirrhosis: results from a retrospective analysis of a large cohort of patients treated at a single center. Endosc Int Open. 2018; 6(3): E266-E270

18. McCarty TR, Rustagi T: Comparative effectiveness and safety of radiofrequency ablation versus argon plasma coagulation for treatment of gastric antral vascular ectasia: A systematic review and meta-analysis. J Clin Gastroenterol. 2018 Jun 26

19. Kar P, Mitra S, Resnick JM, Torbey CF: Gastric Antral Vascular Ectasia: Case Report and Review of the Literature. Clinical Medicine \& Research 2013; 11(2): 80-85.

20. Chiu YC, Lu LS, Wu KL, Tam W, Hu ML, Tai WC et al.: Comparison of argon plasma coagulation in management of upper gastrointestinal angiodysplasia and gastric antral vascular ectasia hemorrhage. BMC Gastroenterol 2012; 12:67.

21. Peter S, Wilcox CM: Radiofrequency ablation therapy - the grave for GAVE (gastric antral vascular ectasia)? Endoscopy International Open 2015; 03: E128-E129

22. Nakamura S, Mitsunaga A, Konishi H, Oi I, Shiratori K, Suzuki S: Long-term follow up of gastric antral vascular ectasia treated by argon plasma coagulation. Dig Endosc 2006; 18: 128-133 
23. Garg S, Aslam B, Nickl N: Endoscopic resolution and recurrence of gastric antral vascular ectasia after serial treatment with argon plasma coagulation. World $J$ Gastrointestinal Endosc. 2017; 9(6):263-266

24. Hermansen JF, Glerup H: Treatment of gastric antral vascular ectasia with endoscopic banding. Ugeskrift for laeger 2015; 177:44

25. Wells CD, Harrison ME, Gurudu SR, Crowell MD, Bryne TJ, Depetris G et al.: Treatment of gastric antral vascular ectasia (watermelon stomach) with endoscopic band ligation. Gastrointest Endosc 2008; 68: 231-236 


\section{Tables}

\begin{tabular}{|c|c|c|c|}
\hline Characteristics & APC $(n=45)$ & EBL $(n=18)$ & p-value \\
\hline Mean age (years) & 66.62 & 68.11 & 0.594 \\
\hline Gender (female/male) & $24 / 21$ & $10 / 8$ & 0.877 \\
\hline \multicolumn{4}{|l|}{ Clinical manifestation } \\
\hline Acute bleeding & $19(42 \%)$ & $5(28 \%)$ & 0.280 \\
\hline Occult bleeding with iron deficiency anemia & $30(67 \%)$ & $13(72 \%)$ & 0.672 \\
\hline \multicolumn{4}{|l|}{ Endoscopic morphology of GAVE } \\
\hline diffuse type & $22(49 \%)$ & $9(50 \%)$ & 0.938 \\
\hline linear type & $23(51 \%)$ & $9(50 \%)$ & 0.938 \\
\hline \multicolumn{4}{|l|}{ Accompanying diseases } \\
\hline liver cirrhosis & $17(38 \%)$ & $10(56 \%)$ & 0.217 \\
\hline hypertension & $28(62 \%)$ & $13(72 \%)$ & 0.450 \\
\hline ischemic heart disease & $15(33 \%)$ & $7(39 \%)$ & 0.690 \\
\hline chronic kidney failure & $3(7 \%)$ & $1(6 \%)$ & 0.869 \\
\hline diabetes mellitus & $21(47 \%)$ & $8(44 \%)$ & 0.877 \\
\hline Raynaud syndrome & $2(4 \%)$ & 0 & 0.160 \\
\hline hypothyroidism & $10(22 \%)$ & $2(11 \%)$ & 0.267 \\
\hline
\end{tabular}

Table 1. Demographic data of patients

GAVE - gastric antral vascular ectasia, APC - argon plasma coagulation, EBL - endoscopic band ligation 
Follow-up period (months)

Total duration of the treatment (months) ${ }^{\dagger}$

Hospitalizations and treatment sessions

Mean number of hospitalizations

Mean number of treatment sessions

Mean number of treatment sessions until

cessation of transfusion need

Mean number of treatment sessions until

endoscopic remission

Mean interval between sessions (weeks) ${ }^{\dagger}$

total

per treatment session
$13.49 \pm 2.46 \quad 18.12 \pm 3.82$

$17.07 \pm 3.74 \quad 9.80 \pm 3.88$

0.185

$4.28 \pm 0.81$

$1.33 \pm 0.33$

$0.002 *$

$5.02 \pm 0.82$

$2.22 \pm 0.45$

$0.004^{*}$

$2.86 \pm 0.68$

$0.90 \pm 0.10$

$0.007^{*}$

$4.43 \pm 0.76$

$1.69 \pm 0.31$

$0.002 *$

$3.75 \pm 0.86$

$3.02 \pm 1.79$

0.719

\section{Change in hemoglobin level (g/dl)}

$2.20 \pm 0.40$

$2.74 \pm 0.61$

0.461

$0.67 \pm 0.19$

$2.13 \pm 0.64$

$0.043^{*}$

\section{Change in required blood transfusions}

(units of packed red blood cells) total

per treatment session

Cessation of transfusion need
$-5.62 \pm 2.30$

$-3.24 \pm 1.94$

25 (56\%)
$-4.79 \pm 2.46$

0.806

$-2.95 \pm 0.92$

0.892

8 (44\%)

0.440

Table 2. Comparison of APC and EBL in the treatment of GAVE syndrome 
GAVE - gastric antral vascular ectasia, APC - argon plasma coagulation, EBL - endoscopic band ligation 


\section{Figure legends}

Figure 1. Argon plasma coagulation (A) and endoscopic band ligation (B)

Figure 2. Diffuse GAVE syndrome treated with argon plasma coagulation (before treatment, after the first and second sessions)

Figure 3. The effect of endoscopic band ligation (before (A) and after (B) treatment)

Figure 4. Changes in hemoglobin level (g/dl) resulting from APC and EBL treatment. Total treatment duration: APC - 17.1 months, EBL -9.8 months $(p=0.185)$

Figure 5. Changes in transfusion need (blood units) resulting from APC and EBL treatment. Total treatment duration: APC - 17.1 months, EBL - 9.8 months $(\mathrm{p}=0.185)$ 

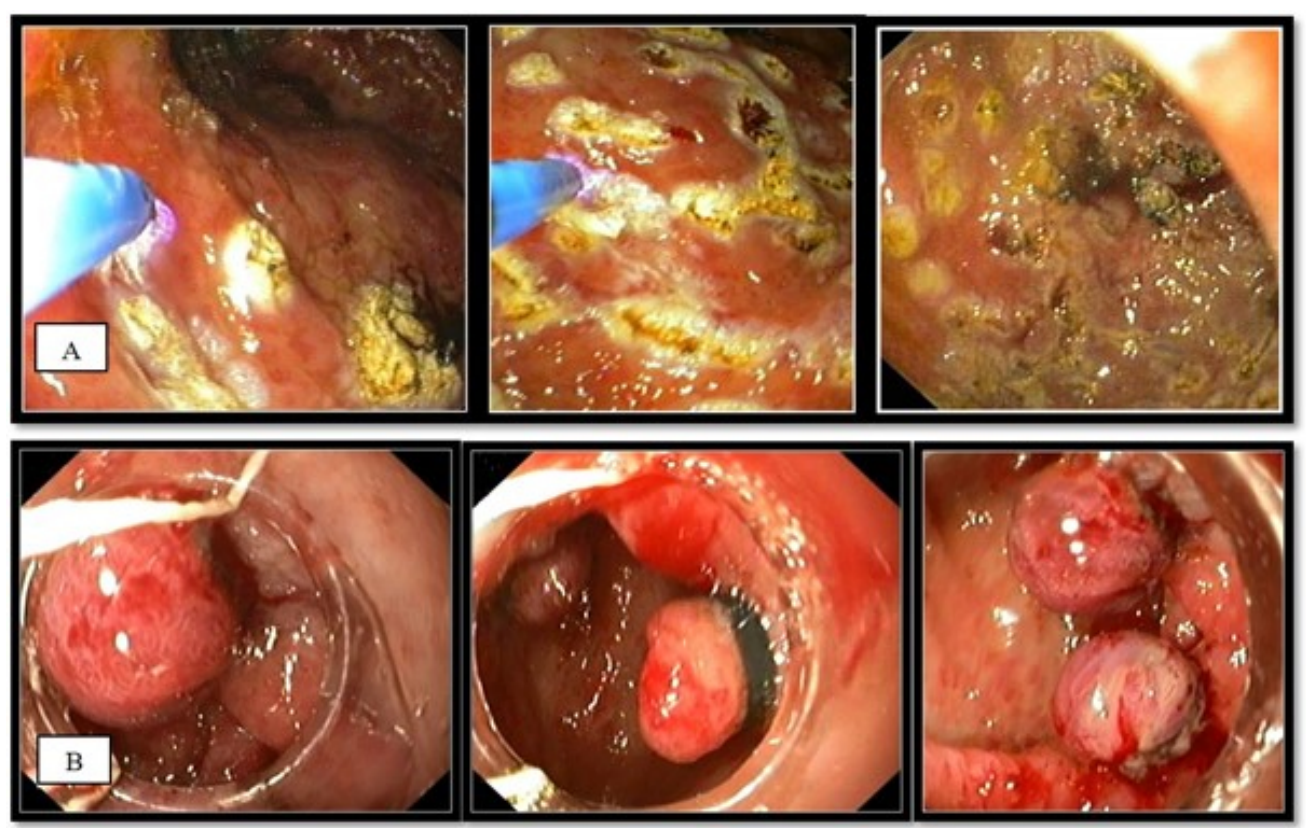

This article is protected by copyright. All rights reserved. 

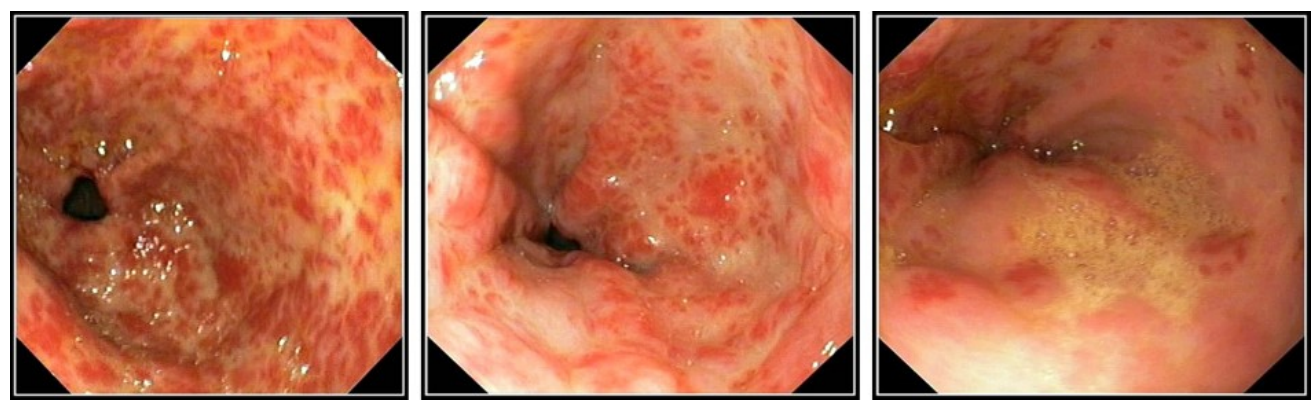

Figure 2. Diffuse GAVE syndrome treated with argon plasma coagulation (before treatment, after the first and second sessions) 

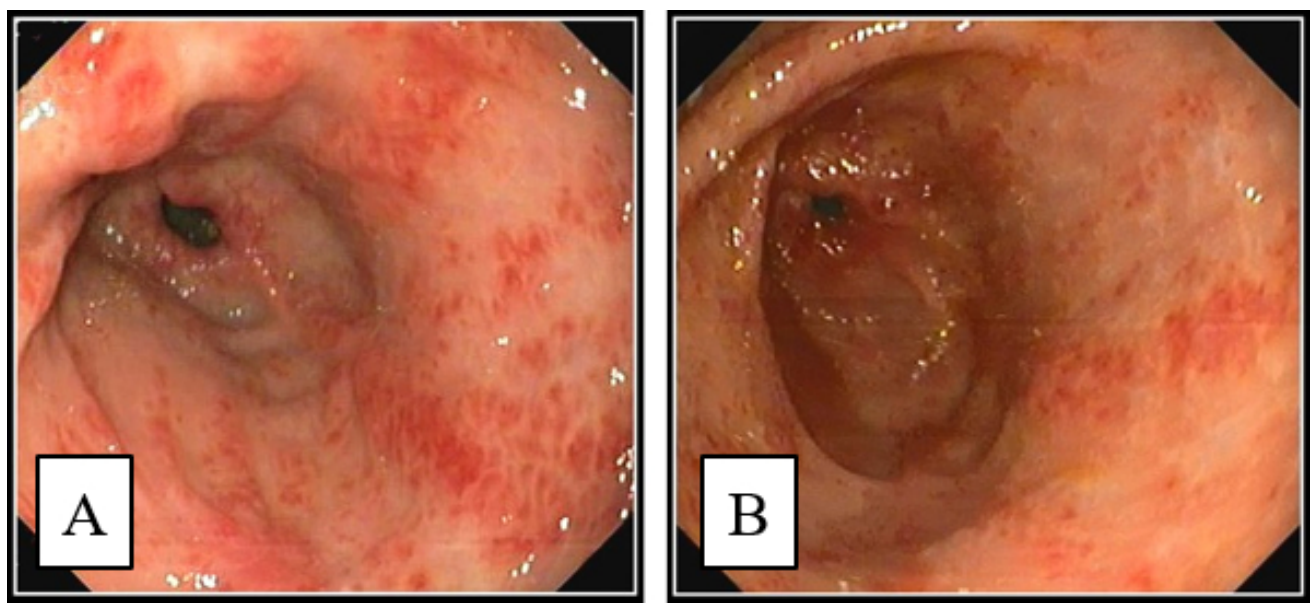

This article is protected by copyright. All rights reserved. 
